\title{
Lasertherapie der Onychomykose: Aktueller Stand
}

\author{
Laser Treatment of Onychomycosis: State of the Art
}

Autoren

Institute
H. Hees ${ }^{1}$, C. Raulin ${ }^{1,2}$

${ }^{1}$ Laserklinik Karlsruhe

${ }^{2}$ Hautklinik der Universität Heidelberg

\section{Bibliografie}

DOI http://dx.doi.org/

10.1055/s-0033-1344197

Akt Dermatol 2013; 39: 278-282

(c) Georg Thieme Verlag KG

Stuttgart · New York

ISSN 0340-2541

\section{Korrespondenzadresse}

Prof. Dr. med. Christian Raulin

Laserklinik Karlsruhe

Kaiserstraße 104

76133 Karlsruhe

info@raulin.de

\section{Zusammenfassung}

$\nabla$

Die Therapie des Nagelpilzes beschränkte sich bislang auf antimykotische Externa und die systemische Therapie. Die zunehmend durchgeführte Lasertherapie könnte die Behandlung der Onychomykose richtungsweisend verändern. Die verfügbaren Lasersysteme werden mit kurzen Behandlungszeiten, Nebenwirkungsfreiheit und

\section{Einführung \\ $\nabla$}

Die Onychomykose ist die am weitesten verbreitete Nagelerkrankung des Erwachsenen [1-3]. Über $10 \%$ der deutschen Bevölkerung sind davon betroffen. Bei über 99\% der Patienten findet sich eine Infektion mit Dermatophyten. Trichophyton rubrum ist dabei für ca. $90 \%$ der Fälle verantwortlich $[4,5]$. Auch ein Befall mit Trichophyton mentagrophytes ist häufig zu finden. Seltene weitere Auslöser der Onychomykose sind Schimmelpilze und Candida-Spezies [6,4]. Die Therapie des Nagelpilzes stellt bis heute eine Herausforderung dar. Sowohl die Anwendung topischer, als auch systemischer Antimykotika ist mit Therapieversagen, langen Behandlungszeiten, hohen Rezidivraten und erheblichen Kosten verbunden [7-11]. Die vielfach praktizierte topische Therapie mit Ciclopirox- oder Amorolfin-Nagellack ist langwierig und führt bei ausgeprägter Onychomykose nur selten zur kompletten Beseitigung des Pilzbefalls [9]. Zudem werden diese Externa von den gesetzlichen Krankenkassen nicht übernommen. Eine in den meisten Fällen zugleich vorhandene Tinea pedum wird außerdem nicht mitbehandelt und ist im Verlauf in aller Regel Reservoir für eine Reinfektion des Nagels. Die systemische Behandlung wird im Allgemeinen mit Terbinafin, Itraconazol oder Fluconazol durchgeführt. Die Intervalltherapie mit einer schematischen Abfolge von Einnahmetagen und Pausen stellt den gegenwärtigen Goldstandard dar [12]. Je nach Ausprägung hoher Effektivität beworben. Nach Zulassung der meisten Geräte durch die FDA (U.S. Food and Drug Administration) steht dem flächendeckenden Einsatz nichts mehr im Wege. Demgegenüber steht eine fehlende Evidenz durch klinische Studien von guter wissenschaftlicher Qualität.

Im Folgenden werden die verfügbaren Geräte samt publizierter Studien zusammenfassend vorgestellt und kritisch diskutiert.

des Befalls ist mit einer Ausheilung nicht vor Ablauf eines Jahres zu rechnen. Vielfach wird von den Patienten eine systemische Therapie über einen so langen Zeitraum abgelehnt oder scheitert an der langfristigen Adhärenz. Auch bei einem vermeintlichen Erfolg der systemischen Behandlung ist von Rezidivraten von etwa $20 \%$ auszugehen [13]. Die Liste unerwünschter Begleitreaktionen und möglicher Interaktionen mit anderen Medikamenten ist bei allen drei Substanzen umfangreich [12,14]. Insbesondere für Patienten mit vorbestehender Leberschädigung und für schwangere oder stillende Frauen fehlt derzeit eine kosteneffiziente, sichere und effektive Alternative.

Die Lasertherapie stellt eine vielversprechende Weiterentwicklung in der Behandlung der Onychomykose dar. Zahlreiche Anbieter haben in den letzten Jahren entsprechende Geräte in ihr Portfolio aufgenommen. Derzeit sind 6 Lasersysteme (Stand Mai 2013) von der amerikanischen Food and Drug Administration (FDA) zur Anwendung bei Pilzinfektionen des Nagelorgans zugelassen. Demgegenüber steht eine denkbar geringe Anzahl nicht immer unabhängig durchgeführter, teils mit systematischen Fehlern behafteten Studien, Fallberichten und Übersichten. Eine Zulassung durch die FDA oder die Betriebsgenehmigung mittels CE-Zeichen bedingen nicht, dass auch randomisierte klinische Studien vorliegen. Die FDA hat sämtliche Lasersysteme aufgrund der Ähnlichkeit mit bereits vorhandenen Geräten 
zur Therapie der Onychomykose zugelassen („substantial equivalence“). Die FDA-Zulassung gilt außerdem nicht wörtlich zur Therapie bzw. Ausheilung der Onychomykose, sondern zum vorübergehenden Nachwachsen klinisch gesunder Nägel („temporary increase of clear nail“) $[15,16]$. In Deutschland gilt die Zulassung durch Kennzeichnung mit dem CE-Zeichen ohne genauere Differenzierung. Die zunächst für Verwirrung sorgende Situation lässt sich durch systematische Gliederung und wissenschaftliche Analyse der vorhandenen Publikationen ordnen. Dies ist das Ziel der vorliegenden Übersicht.

\section{Wirkmechnismus}

$\nabla$

Der genaue Wirkmechanismus des Laserlichts auf mykotisches Material wird mit verschiedenen Hypothesen diskutiert und ist bislang ungeklärt. Wir favorisieren, wie die meisten Autoren, thermische Effekte als Hauptfaktoren der Wirksamkeit. Bei der selektiven Photothermolyse resultiert die Absorption der Energie des Lasers durch Pilzmaterial in der Entstehung von Hitze und mechanischer Energie [17]. Pilzmyzel ist außerordentlich kältestabil, aber hitzeempfindlich; Temperaturen über 50 Grad Celsius für mehrere Minuten wirken letal auf vorhandenes mykotisches Gewebe $[18,19]$. Die Laserenergie muss möglichst selektiv thermisch auf das Pilzmyzel einwirken, in eine bestimmte Tiefenebene eindringen und dabei die umgebenden Strukturen der Haut schonen. Dies erlauben Nd:YAG-Laser mit kurzer Impulsdauer [25]. Die Photothermolyse ist wahrscheinlich nicht allein verantwortlich für die Effekte der Lasertherapie. Diskutiert werden daneben die Beeinflussung intrazellulärer Redox-Reaktionen bei der Verwendung von langgepulsten Systemen mit Wellenlängen im nahen Infrarotbereich [20]. Hierbei könnten die Erhöhung des Spiegels reaktiver Sauerstoffspezies und die Verringerung des Membranpotentials der Mitochondrien antimykotische Effekte erzielen. Ablative und thermische Wirkungen sind hier nicht zu erwarten. Außerdem könnte eine unspezifische Erwärmung des Gewebes mit folgender Durchblutungssteigerung durch Vasodilatation und Stimulierung von immunologischen Prozessen für die Wirkung mitverantwortlich sein [15].

\section{Studienlage}

$\nabla$

Die kommerziell verfügbaren Systeme sind überwiegend $\mathrm{Nd}$ : YAG-Laser, sowohl im Milli- und Mikrosekundenbereich (langund kurzgepulst) als auch im Nanosekundenbereich (gütegeschaltet, q-switched) [21]. Daneben wurden zwei Diodenlaser sowie ein Titan:Saphir-Laser in Studien untersucht.

Manevitch et al. konnten in einer in vitro Studie nach einmaliger Behandlung von mykotisch veränderten Nägeln mit einem femtosekundengepulsten Titan:Saphir-Laser (Coherent Mira ${ }^{\circledR}$ 900, $780-820 \mathrm{~nm}$ ) nach 4 Wochen kein Wachstum mehr in einer direkt danach angelegten Pilzkultur feststellen [22]. Ob diese Ergebnisse in vivo zu reproduzieren sind, ist unklar. Wegen der enorm hohen Anschaffungskosten werden Femtosekundenlaser bislang nur im experimentellen Bereich eingesetzt. Daher ist diesem Lasersystem aktuell keine Bedeutung in der Therapie der Onychomykose beizumessen.

\section{Diodenlaser}

Zwei Diodenlaser befinden sich derzeit in der klinischen Evaluation. Der Noveon ${ }^{\circledR}$-Laser (Nomir Medical Technologies) kombiniert die Wellenlängen $870 \mathrm{~nm}$ und $930 \mathrm{~nm}$. In einer klinischen Studie von Landsman et al. an 26 befallenen Fußnägeln ergaben sich nach 4 Behandlungen (Tag 1, 14, 42 und 120) in 30\% der Fälle negative Pilzkulturen. Auch nach 270 Tagen war dieser Befund reproduzierbar $[23,24]$. Die Wellenlängen in der Nähe des Infrarotbereichs erzeugen im Vergleich zum Nd:YAG-Laser mit $1064 \mathrm{~nm}$ keine thermische Energie und reduzieren somit potenziell schädigende Effekte am umgebenden Gewebe [20]. Es sind 2 weitere unveröffentlichte Studien beim amerikanischen Gesundheitsministerium mit derzeit unbekanntem Status registriert (clinical trials identifier: NCT00776464). Der V-Raser ${ }^{\circledR}(980 \mathrm{~nm}$, ConBio/Cynosure) befindet sich derzeit ebenfalls in der klinischen Evaluation (NCT01452490). Der genaue Wirkmechanismus bleibt ungeklärt und es sind vor der klinischen Anwendung unabhängige klinische Studien zu fordern. Daher kommt auch dem Diodenlaser derzeit in der Therapie der Onychomykose eine eher untergeordnete Rolle $\mathrm{zu}$.

\section{Nd:YAG-Laser}

Den Nd:YAG-Lasern mit einer Wellenlänge von $1064 \mathrm{~nm}$ kommt im Hinblick auf die therapeutische Wirksamkeit und die vorhandene Evidenz die größte Bedeutung zu. Die meisten veröffentlichten Studien untersuchen die Wirksamkeit dieser Systeme. Sie sind bereits für viele Indikationen etabliert und vergleichsweise wenig wartungsanfällig. Tab. 1 gibt einen Überblick über die derzeit erhältlichen Nd:YAG-Laser. Tab. 2 fasst die unten genannten Veröffentlichungen zusammen.

Langgepulste Nd:YAG-Laser benötigen zur Applikation von genügend Energie eine Kaltluftkühlung [25], um umliegende Strukturen, die nicht das Target darstellen, zu schonen. Unerwünschte thermische Wirkungen auf das kollaterale Gewebe stellen einen Nachteil der langen Pulsdauer dar. Zwei Arbeitsgruppen haben jeweils einen langgepulsten Nd:YAG-Laser mit 1064 nm Wellenlänge klinisch untersucht. Kozarev et al. behandelten 72 Patienten mit einer Energiedichte von $35-40 \mathrm{~J} / \mathrm{cm}^{2}, 35 \mathrm{~ms}$ Pulsdauer, $4 \mathrm{~mm}$ Spot, 3 Durchgängen und 4 Sitzungen im Abstand von je einer Woche [26]. Zhang et al. verwendeten bei 33 Patienten die Parameter $230-324 \mathrm{~J} / \mathrm{cm}^{2}, 30 \mathrm{~ms}, 3 \mathrm{~mm}, 4$ oder 8 Sitzungen im Abstand von einer Woche [27]. Beide konnten mit 50\% negativen Pilzkulturen bzw. nahezu 100\% klinisch-makroskopischer Ausheilung klare Erfolge beschreiben. Die Veröffentlichung von Kozarev erschien in einer Zeitschrift ohne Impact Faktor und hatte methodische Mängel. Wir konnten diese Ergebnisse in einer eigenen In-vitro-Pilotstudie leider nicht reproduzieren. Zhang et al. setzten vermeintlich den PinPointe ${ }^{\mathrm{TM}}$ Footlaser $^{\mathrm{TM}}$ ein. Dies ist jedoch in keiner Weise mit den angegeben technischen Daten in Einklang zu bringen, was auch vom Hersteller des Lasers so bewertet wird. Beide Studien entsprechen nicht den geforderten wissenschaftlichen Standards. Ihre Ergebnisse sind daher nur sehr eingeschränkt zu werten und werden unter diesen besonderen Gesichtspunkten diskutiert.

Die Wirksamkeit des kurzgepulsten Nd:YAG-Lasers wird in 3 klinischen Studien beschrieben. In einem White Paper von Weiss et al. mit 7 Patienten konnten nach 2 Behandlungen mit einem $1064 \mathrm{~nm}$ Nd:YAG-Laser (Genesis plus ${ }^{\mathrm{TM}}$, Cutera) $70 \%$ der Nägel nach 12 Wochen klinisch gebessert werden [28]. Die geringe Patientenzahl und die unvollständige statistische Aufarbeitung der Ergebnisse lässt keine endgültige Aussage zur Wirksamkeit zu. Eine japanische Studie untersuchte ebenfalls einen kurzgepuls- 
Tab. 1 Nd:YAG-Laser zur Therapie der Onychomykose.

\begin{tabular}{|c|c|c|c|c|}
\hline System & Typ, Wellenlänge (nm) & Energiedichte $\left(\mathrm{J} / \mathrm{cm}^{2}\right)$ & Impulsdauer & Spotgröße (mm) \\
\hline $\begin{array}{l}\text { PinPointe }^{\mathrm{TM}} \\
\text { Footlaser }^{\mathrm{TM}} \\
\text { Nuvolase }\end{array}$ & kurzgepulster Nd:YAG $1064 \mathrm{~nm}$ & 25,5 & $0,1 \mathrm{~ms}$ & $1-1,5$ \\
\hline $\begin{array}{l}\text { GenesisPlus' }{ }^{\mathrm{M}} \text {, } \\
\text { Cutera }\end{array}$ & kurzgepulster Nd:YAG $1064 \mathrm{~nm}$ & $5-40$ & $0,1-1 \mathrm{~ms}$ & $1-1,5$ \\
\hline $\begin{array}{l}\text { Fotona XP'MLine, } \\
\text { Fotona }\end{array}$ & kurzgepulster Nd:YAG $1064 \mathrm{~nm}$ & $\max .3,5$ & $0,1-1 \mathrm{~ms}$ & $1-1,5$ \\
\hline $\begin{array}{l}\text { Joule } \\
\text { ClearSense }{ }^{\mathrm{M}} \text {, } \\
\text { Sciton }\end{array}$ & kurzgepulster Nd:YAG $1064 \mathrm{~nm}$ & 25,5 & $0,1-200 \mathrm{~ms}$ & $1-1,5$ \\
\hline $\begin{array}{l}\text { VARIA } \\
\text { CoolTouch }\end{array}$ & kurzgepulster Nd:YAG $1064 \mathrm{~nm}$ & - & $0,6 \mathrm{~ms}$ & - \\
\hline $\begin{array}{l}\text { Q-Clear }{ }^{T M} \\
\text { Light Age }\end{array}$ & gütegeschalteter Nd:YAG 1064 nm & $\max .0,6$ & $3-10 n s$ & 2,5 \\
\hline $\begin{array}{l}\text { LightPod }^{\circledR} \\
\text { Neo }^{T M *} \text {, } \\
\text { Aerolase }\end{array}$ & kurzgepulster Nd:YAG $1064 \mathrm{~nm}$ & 223 & $0,65 \mathrm{~ms}$ & 2 \\
\hline $\begin{array}{l}\text { CT3 Plus }{ }^{\mathrm{TM} *} \text {, } \\
\text { CoolTouch }\end{array}$ & kurzgepulster Nd:YAG $1320 \mathrm{~nm}$ & - & $0,45 \mathrm{~ms}$ & $2-10$ \\
\hline $\begin{array}{l}\text { Dualis SPтM* } \\
\text { Fotona }\end{array}$ & langgepulster Nd:YAG $1064 \mathrm{~nm}$ & $35-40$ & $35 \mathrm{~ms}$ & 4 \\
\hline
\end{tabular}

* keine FDA-Zulassung

Tab.2 Studien zur Wirksamkeit des 1064 nm-Nd:YAG-Lasers (Stand 4/2013).

\begin{tabular}{|c|c|c|c|c|c|}
\hline Autor & Laser & $\begin{array}{l}\text { Parameter } \\
\text { (Energiedichte, Impulsdauer, } \\
\text { Spotgröße, Wiederholungsrate) }\end{array}$ & Patienten (n) & Follow-up & Ergebnis \\
\hline Weiss et al. & $\begin{array}{l}\text { Genesis plus }{ }^{\top M} \text {, } \\
\text { Cutera }\end{array}$ & $\begin{array}{l}16 \mathrm{~J} / \mathrm{cm}^{2}, 0,3 \mathrm{~ms}, 5 \mathrm{~mm}, 2 \mathrm{~Hz}, \\
2 \text { Beh. Abstand } 6 \text { Wo }\end{array}$ & 7 & 6,12 Wo & $\begin{array}{l}\text { Verbesserung in } 70 \% \\
\text { der Fälle }\end{array}$ \\
\hline Kozarev et al. & Dualis SPTM, Fotona & $\begin{array}{l}35-40 \mathrm{~J} / \mathrm{cm}^{2}, 35 \mathrm{~ms}, 4 \mathrm{~mm}, 1 \mathrm{~Hz} \\
3 \text { passes, } 4 \text { Beh. } 1 \text { Wo Abstand }\end{array}$ & 72 & $3,6,9,12 \mathrm{Mo}$ & $100 \%$ Clearance \\
\hline Kimura et al. & $\begin{array}{l}\text { Xeo }{ }^{\mathrm{TM}} \text { Laser Genesis }{ }^{\mathrm{TM}} \text {, } \\
\text { Cutera }\end{array}$ & $\begin{array}{l}14 \mathrm{~J} / \mathrm{cm}^{2}, 0,3 \mathrm{~ms}, 5 \mathrm{~mm}, 5 \mathrm{~Hz} \text {, } \\
2 \text { passes, } 1-3 \text { Beh., } 4-8 \text { Wo Abstand }\end{array}$ & 13 & $1,2,3,4,5,6 \mathrm{Mo}$ & $\begin{array}{l}\text { in } 50 \% \text { komplette } \\
\text { Clearance }\end{array}$ \\
\hline Zhang et al. & 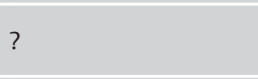 & $\begin{array}{l}240-324 \mathrm{~J} / \mathrm{cm}^{2}, 30 \mathrm{~ms}, 3 \mathrm{~mm}, 1 \mathrm{~Hz} \text {, } \\
4 \text { und } 8 \text { Sitzungen, Abstand } 1 \mathrm{Wo}\end{array}$ & 33 & $2,4,6 \mathrm{Mo}$ & $\begin{array}{l}\text { Rund } 50 \% \text { mykol. } \\
\text { Clearance nach } 6 \text { Mo }\end{array}$ \\
\hline Hochman et al. & $\begin{array}{l}\text { LightPod } \mathrm{Neo}^{\mathrm{TM}} \text {, } \\
\text { Aerolase }\end{array}$ & $\begin{array}{l}223 \mathrm{~J} / \mathrm{cm}^{2}, 0,65 \mathrm{~ms}, 2 \mathrm{~mm}, \\
2-3 \text { Sitzungen, Abstand } 3 \text { Wo }\end{array}$ & 8 & 4-6 Mo & $\begin{array}{l}7 \text { von } 8 \text { Pat. mykol. } \\
\text { Clearance }\end{array}$ \\
\hline
\end{tabular}

ten Nd:YAG-Laser mit 1064 nm Wellenlänge (Cutera Xeo ${ }^{\mathrm{TM}}$ Plattform, Laser Genesis ${ }^{\mathrm{TM}}$ ähnlich Genesis plus ${ }^{\mathrm{TM}}$, größerer Strahldurchmesser von $5 \mathrm{~mm}$, nicht in $\bullet$ Tab. 1 aufgeführt). In der Arbeit mit 13 Probanden konnte nach 1-3 Behandlungen mit 4-8 Wochen Abstand in 50\% der Fälle eine komplette klinische Ausheilung und negative Pilzkultur 16 Wochen nach der letzten Behandlung erzielt werden [29]. Die Veröffentlichung lässt aufgrund der kurzen Nachbeobachtungszeit von nur 16 Wochen keine Aussage über die längerfristige Wirksamkeit zu. Eine längere Nachbeobachtungszeit von 4-6 Monaten ist in einer Studie von Hochman et al. zu finden. Nach 2 - 3 Sitzungen mit einem kurzgepulsten Nd:YAG-Laser (LightPod $\mathrm{Neo}^{\mathrm{TM}}$, Aerolase) im Abstand von 3 Wochen hatten 7 von 8 untersuchten Patienten (11 von 12 Nägeln) eine negative Pilzkultur [25] zu verzeichnen. Dieser Laser war bis zum Zeitpunkt der Drucklegung nicht von der FDA zur Nagelpilzbehandlung zugelassen.

\section{PinPointe ${ }^{\mathrm{TM}}$ Footlaser $^{\mathrm{TM}}$}

Zur Wirksamkeit des PinPointe ${ }^{\mathrm{TM}}$ Footlaser ${ }^{\mathrm{TM}}$ (Nuvolase, Cynosure) liegen bislang nur White Papers, allerdings noch keine veröffentlichte klinische Studie mit peer-review vor. Eine Untersu- chung unserer Arbeitsgruppe steht kurz vor der Fertigstellung. Der Vorteil dieses kurzgepulsten Nd:YAG-Lasers liegt in der Möglichkeit deutlich mehr Energie pro Fläche abzugeben, ohne dabei das umliegende Gewebe thermisch zu schädigen, was für den Patienten schmerzhaft wäre. Der PinPointe ${ }^{\mathrm{TM}}$ Footlaser $^{\mathrm{TM}}$ appliziert als Gesamtimpuls (Makropuls) eine Serie von 10 Mikropulsen mit einer Pulsdauer von je 0,1 ms und einer Energiedichte von je $20 \mathrm{~J} / \mathrm{cm}^{2}$. Ein Makropuls umfasst $500 \mathrm{~ms}$, womit zwischen den einzelnen Mikropulsen eine Pause von $50 \mathrm{~ms}$ liegt. Der besondere Vorteil bei der Therapie der Onychomykose ist die vergleichsweise hohe Energiedichte von insgesamt $200 \mathrm{~J} / \mathrm{cm}^{2}$, welche pro Makropuls abgegeben wird. Diese Energiedichte wird nur deshalb vom Patienten toleriert, weil zwischen den einzelnen Mikropulsen Pausen von $50 \mathrm{~ms}$ liegen. Diese sind länger als die thermische Relaxationszeit von Gefäßen und Nerven im umliegenden Gewebe, wodurch eine Abkühlung dieser innerhalb eines Impulses gewährleistet ist. Die thermische Relaxationszeit der mykotischen Zielstrukturen unterhalb und innerhalb des Nagels ist jedoch deutlich länger als $50 \mathrm{~ms}$, wodurch hier thermische Energie kummuliert und antimykotische Temperaturen erreicht werden. 


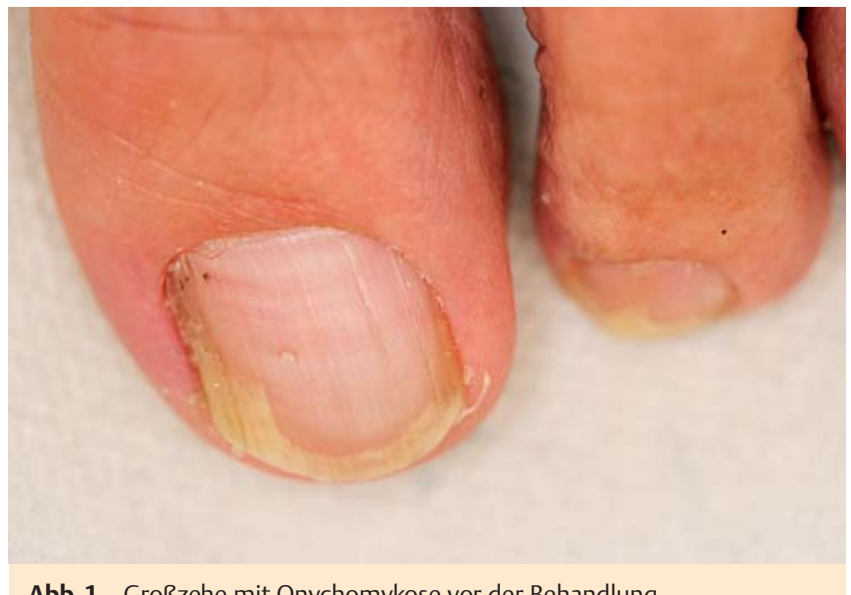

Abb.1 Großzehe mit Onychomykose vor der Behandlung.

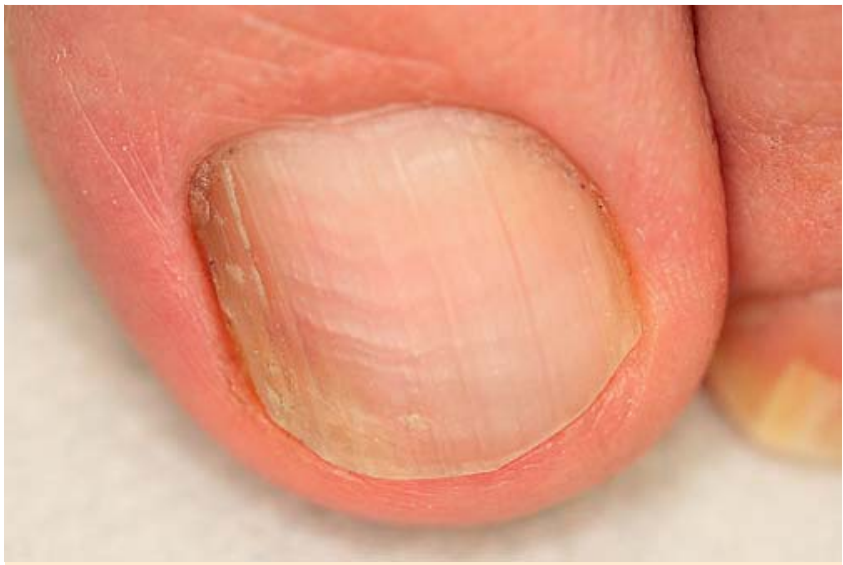

Abb. 2 Zustand 3 Monate nach 2 Sitzungen mit dem PinPointe ${ }^{\mathrm{TM}}$ Footlaser ${ }^{\mathrm{TM}}$.

Wir führten eine randomisierte „split-feet“ Studie an 15 Probanden mit histologisch und kulturell gesicherter Onychomykose der Großzehennägel durch. Die Behandlung erfolgte an den beiden Großzehen mit dem PinPointe ${ }^{\mathrm{TM}}$ Footlaser $^{\mathrm{TM}}$ und einem langgepulsten Nd:YAG-Laser („Elite“, Cynosure Inc., USA) mit 1064 nm Wellenlänge, $50 \mathrm{~J} / \mathrm{cm}^{2}$ Energiedichte, $40 \mathrm{~ms}$ Impulsdauer und $3 \mathrm{~mm}$ Spotgröße. Es erfolgten jeweils 4 Behandlungsdurchgänge und zwei Lasersitzungen im Abstand von 2 Wochen. Die Patienten behandelten die Umgebung zur Rezidivprophylaxe ergänzend mit einer antimykotischen Creme. Die bislang unveröffentlichten Rohdaten lassen eine positive Aussage zur Wirksamkeit beider Lasersysteme zu. Die Ergebnisse sind jedoch individuell unterschiedlich. $\bullet$ Abb. 1 und $\bullet$ Abb. 2 zeigen positive Beispiele der Wirksamkeit des PinPointe ${ }^{\mathrm{TM}}$ Footlaser $^{\mathrm{TM}}$. Laut Herstellerprospekt soll eine Behandlung ausreichen. Wir können das in dieser Form nicht bestätigen. Patienten, die in unserem Zentrum außerhalb der Studie behandelt werden, empfehlen wir unter der Vorstellung einer eher defensiven Beratung und Aufklärung etwa 6 Behandlungen in 2- 6 wöchigen Abständen mit ansprechenden Resultaten. Laut Nuvolase/Cynosure ist die Behandlung schmerzfrei. Wir sehen auch dies etwas differenzierter. Bei Applikation von 4 Laserdurchgängen äußert der Patient je nach Nageldicke deutliche, wenn auch kurz anhaltende Schmerzen, die aber in aller Regel gut toleriert werden. Diese sind für den Behandler ein richtungsweisendes Zeichen für das Erreichen der gewünschten Zieltemperatur. Keiner unserer Patienten hat die Therapie bislang deshalb abgebrochen.

\section{Kombinationstherapie und rechtliche Situation}

Für jede Art der Lasertherapie der Onychomykose gilt es zu bedenken, dass lediglich der Nagel behandelt wird. Eine meist zugleich vorhandene ausgeprägte aber oftmals vom Patienten nicht realisierte Tinea pedum ist sicheres Reservoir für ein Rezidiv. Die insbesondere von den Laserherstellern propagierte Monotherapie scheidet somit in den meisten Fällen aus. Es empfiehlt sich deshalb aus unserer Sicht die Kombinationstherapie aus systemischem Antimykotikum oder einer mehrmonatigen Lokaltherapie der Tinea pedum in Verbindung mit einer Laserbehandlung der Nägel. Der wesentliche Vorteil der begleitenden Lasertherapie liegt bei Kombination mit einem oralen Antimykotikum dann in der deutlich verkürzten Einnahmedauer des Medikaments. Unserer klinischen Erfahrung nach kann durch die Laseranwendungen des PinPointe ${ }^{\mathrm{TM}}$ Footlaser $^{\mathrm{TM}}$ eine systemische Behandlung auf die Hälfte der Zeit reduziert werden.

Im Hinblick auf den Einsatz der Lasertherapie bei Onychomykose ist das Urteil des Verwaltungsgerichtes Arnsberg vom 8. Mai 2012 bedeutsam. Die Behandlung einer Nagelpilzinfektion mittels Laser wird darin eindeutig als Ausübung der Heilkunde angesehen. Somit ist es nur Ärztinnen und Ärzten, sowie medizinischen Heilberufen nach dem Heilpraktikergesetz (HeilprG) gestattet, eine solche Therapie gewerblich durchzuführen. Podologen ist die Lasertherapie durch diesen Gerichtsbeschluss explizit untersagt [30].

\section{Zusammenfassung}

Der Laser muss seinen Stellenwert als zusätzliche Behandlungsoption der Onychomykose noch finden. Das Interesse an einer neuen effektiven Therapieform ist zweifelsohne enorm groß. Eine undifferenzierte Euphorie ist jedoch zum jetzigen Zeitpunkt noch nicht angebracht. Die von den Herstellern verbreiteten Erfolgsmeldungen sind nur unter Vorbehalt zu sehen und viele der beworbenen Vorteile unterliegen bei genauer kritischer Betrachtung deutlichen Einschränkungen.

Die bisher veröffentlichten Studien erfüllen unserer Einschätzung nach aus Gründen des Studienaufbaus, der zu geringen Patientenzahl, dem Grundsatz der eingeschränkten Objektivität und einem zu kurzen Nachbeobachtungszeitraum, nicht die Kriterien, die für eine kritische evidenzbasierte Publikation zu fordern wären. Darüber hinaus müssen die Patienten darüber aufgeklärt werden, dass die Behandlung oftmals unangenehm ist, dass sehr wahrscheinlich mehrere Sitzungen erforderlich sind, und dass in den meisten Fällen eine Kombinationstherapie mit einem systemischen oder zumindest einem lokalen Antimykotikum bei komorbider Tinea pedum notwendig ist. Bei Beachtung dieser Einschränkungen ist die Anwendung des Lasers zur Therapie der Onychomykose sinnvoll und bei richtiger Diagnosestellung und Wahl eines geeigneten Gerätes mit seriös evaluiertem Wirkungsnachweis zukunftsweisend und erfolgversprechend. 


\section{Interessenkonflikt}

$\nabla$

Dem korrespondierenden Autor wurde seitens Cynosure ein PinPointe ${ }^{\mathrm{TM}}$ Footlaser ${ }^{\mathrm{TM}}$ für die Dauer von 6 Monaten zur eigenen Meinungsbildung zur Verfügung gestellt.

\section{Abstract}

\section{Laser Treatment of Onychomycosis. State of the Art}

Antimycotic ointment and systemic therapy have been the state of the art treatment of nail fungus for years. Since laser therapy has recently been performed on a larger scale, it could give direction to onychomycosis treatment in the future. The available laser systems are advertised with considerable advantages: short time of treatment, no adverse effects, complete nail clearance after a few sessions. Most of the devices are FDA (U.S. Food and Drug Administration) approved but the lack of evidence and clinical trials that were published in influential journals tarnish the positive image.

In this overview available laser devices and published research studies are presented and discussed in a critical manner.

\section{Literatur}

1 Ghannoum MA, Hajjeh RA, Scher $R$ et al. A large-scale North American study of fungal isolates from nails: the frequency of onychomycosis, fungal distribution, and antifungal susceptibility patterns. J Am Acad Dermatol 2000; 43: 641-648

2 Schlefman BS. Onychomycosis: a compendium of facts and a clinical experience. J Foot Ankle Surg 1999; 38: 290-302

3 Drake LA, Patrick DL, Fleckman P et al. The impact of onychomycosis on quality of life: development of an international onychomycosis-specific questionnaire to measure patient quality of life. J Am Acad Dermatol 1999; 41: 189-196

4 Evans EG. Causative pathogens in onychomycosis and the possibility of treatment resistance: a review. J Am Acad Dermatol 1998; 38: 32 - 36

5 Zaias N, Glick B, Rebell G. Diagnosing and treating onychomycosis. J Fam Pract 1996; 42: 513-518

6 Gupta AK, Drummond-Main C, Cooper EA et al. Systematic review of nondermatophyte mold onychomycosis: Diagnosis, clinical types, epidemiology, and treatment. J Am Acad Dermatol 2012; 66: 494-502

7 Albert SF, Weis ZH. Management of onychomycosis with topicals. Clin Podiatr Med Surg 2004; 21: 605-615 vii

8 Bodman MA, Feder L, Nace AM. Topical treatments for onychomycosis: a historical perspective. J Am Podiatr Med Assoc 2003; 93: 136-141

9 Brenner MA, Harkless LB, Mendicino RW et al. Ciclopirox 8\% nail lacquer topical solution for the treatment of onychomycosis in patients with diabetes: a multicenter, open-label study. J Am Podiatr Med Assoc 2007; 97: 195-202

10 Tosti A, Piraccini BM, Stinchi C et al. Relapses of onychomycosis after successful treatment with systemic antifungals: a three-year followup. Dermatology 1998; 197: 162 - 166
11 Finch JJ, Warshaw EM. Toenail onychomycosis: current and future treatment options. Dermatol Ther 2007; 20: 31 - 46

12 De Doncker P, Decroix J, Pierard GE et al. Antifungal pulse therapy for onychomycosis. A pharmacokinetic and pharmacodynamic investigation of monthly cycles of 1 -week pulse therapy with itraconazole. Arch Dermatol 1996; 132: $34-41$

13 Piraccini BM, Sisti A, Tosti A. Long-term follow-up of toenail onychomycosis caused by dermatophytes after successful treatment with systemic antifungal agents. J Am Acad Dermatol 2010; 62: 411 - 414

14 Gupta AK, Uro M, Cooper EA. Onychomycosis therapy: past, present, future. J Drugs Dermatol 2010; 9: 1109-1113

15 Gupta A, Simpson F. Device-based therapies for onychomycosis treatment. Skin Therapy Lett 2012; 17: 4-9

16 Gupta AK, Simpson F. Newly approved laser systems for onychomycosis. J Am Podiatr Med Assoc 2012; 102: 428 - 430

17 Altshuler GB, Anderson RR, Manstein D et al. Extended theory of selective photothermolysis. Lasers Surg Med 2001; 29: 416-432

18 Emyanitoff $R G$, Hashimoto $T$. The effects of temperature, incubation atmosphere, and medium composition on arthrospore formation in the fungus Trichophyton mentagrophytes. Can J Microbiol 1979; 25: $362-366$

19 Hashimoto T, Blumenthal HJ. Survival and resistance of Trichophyton mentagrophytes arthrospores. Appl Environ Microbiol 1978; 35: 274-277

20 Bornstein E, Hermans W, Gridley S et al. Near-infrared photoinactivation of bacteria and fungi at physiologic temperatures. Photochem Photobiol 2009; 85: 1364-1374

21 Raulin C, Kimmig W, Werner S. [Laser therapy in dermatology and esthetic medicine. Side effects, complications and treatment errors]. Hautarzt 2000; 51: $463-473$

22 Manevitch $Z$, Lev D, Hochberg $M$ et al. Direct antifungal effect of femtosecond laser on Trichophyton rubrum onychomycosis. Photochem Photobiol 2009; 86: 476-479

23 Landsman AS, Robbins AH. Treatment of Mild, Moderate, and Severe Onychomycosis Using 870- and 930-nm Light Exposure: Some Follow-up Observations at 270 Days. J Am Podiatr Med Assoc 2012; 102: $169-171$

24 Landsman AS, Robbins AH, Angelini PF et al. Treatment of mild, moderate, and severe onychomycosis using 870 - and 930-nm light exposure. J Am Podiatr Med Assoc 2010; 100: 166-177

25 Hochman LG. Laser treatment of onychomycosis using a novel 0.65 millisecond pulsed Nd:YAG 1064-nm laser. J Cosmet Laser Ther 2011; 13: $2-5$

26 Kozarev J, Vizintin Z. Novel laser therapy in treatment of onychomycosis. Journal of the Laser and Health Academy 2010: 1-8

27 Zhang RN, Wang DK, Zhuo FL et al. Long-pulse Nd:YAG 1064-nm laser treatment for onychomycosis. Chin Med J (Engl) 2012; 125: 3288 3291

28 Weiss D. 3 month clinical results using sub-millisecond $1064 \mathrm{~nm} \mathrm{Nd:}$ YAG laser for the treatment of oynchomycosis. Available at: http://conejofeet.com/wp-content/uploads/2011/06/David-Weiss-DPM-report.pdf. Dem Internet entnommen im August 2012.

29 Kimura U, Takeuchi K, Kinoshita A et al. Treating Onychomycoses of the Toenail: Clinical Efficacy of the Sub-Millisecond 1,064nm Nd:YAG Laser Using a $5 \mathrm{~mm}$ Spot Diameter. J Drugs Dermatol 2012; 11: 496 504

30 VwG Arnsberg. Beschl. vom 8.5.2012. 3L247/12 